\title{
3-Methylglutaconic Aciduria
}

National Cancer Institute

\section{Source}

National Cancer Institute. 3-Methylg/utaconic Aciduria. NCI Thesaurus. Code C98678.

A group of five inherited disorders caused by mutations in the AUH, DNAJC19, OPA3, and

TAZ genes. The disorders are characterized by impairment in the function of

mitochondria, resulting in the accumulation and excretion of 3-methylg lutaconic acid, and the presence of 3-methylglutaric acid in the urine. 